\title{
Case of a Giant Conjunctival Melanocytic Nevus
}

\author{
Hidetsugu Mori Kanji Takahashi \\ Department of Ophthalmology, Kansai Medical University, Osaka, Japan
}

\section{Established Facts}

- When giant conjunctival melanocytic masses are confirmed, most cases must be suspected to be conjunctival malignant melanoma.

\section{Novel Insights}

- We describe an unusual case of a giant conjunctival melanocytic nevus.

\section{Keywords}

Giant conjunctival melanocytic nevi · Conjunctival malignant melanoma

\section{Abstract \\ A 78-year-old female experienced extraocular extension of a giant conjunctival melanocytic mass. The clinical diagnosis was conjunctival malignant melanoma. We performed local excision and histopathological examination. The result of hematoxylin-eosin staining disclosed multiple intralesional mucosal cysts and nevus cell nests with abundant melanin. Immunohistochemical examination revealed expression of S-100, melan-A, and HMB-45 and no expression of Ki-67. His- topathological examination showed no evidence of malig- nancy. Giant conjunctival melanocytic nevi can be diagnosti- cally confused with conjunctival malignant melanoma.}

(c) 2020 S. Karger AG, Basel

\section{Introduction}

Conjunctival melanocytic nevi are the most common type of conjunctival benign tumor $[1,2]$. Most observed conjunctival melanocytic nevi are small $[1,2]$; conjunctival nevi $\geq 10 \mathrm{~mm}$ in basal diameter are called "giant" conjunctival nevi [3]. In the case of large conjunctival melanocytic tumors, it is difficult to distinguish between conjunctival malignant melanoma and giant conjunctival melanocytic nevi clinically [3-5]. Herein, we describe a rare case of giant conjunctival melanocytic nevus that was suspected to be conjunctival malignant melanoma clinically and diagnosed as benign by histopathological examination.

\section{Case Report}

A 78-year-old Japanese female was referred to Kansai Medical University Hospital in March 2019 with a complaint of a large, darkbrown mass located on the right lower eyelid. In her childhood, a 

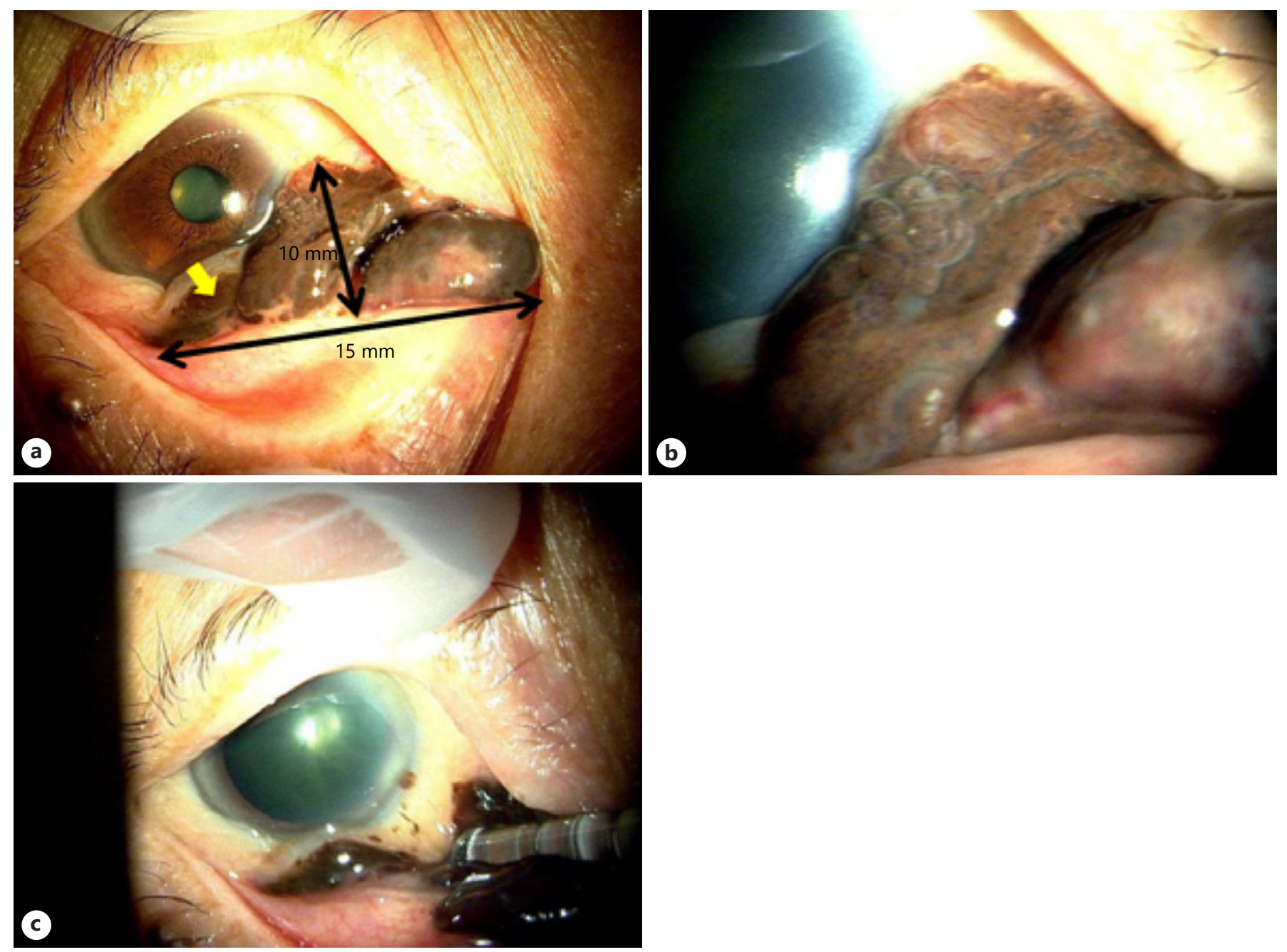

Fig. 1. Initial right extraocular findings. a A pigmented mass of 10 to $15 \mathrm{~mm}$ in diameter protruding out of the lower eyelid of the right eye showed invasion of the inferior conjunctival fornix (yellow arrow). $\mathbf{b}$ The giant mass demonstrated an irregular surface and no intralesional cysts. c Pigment deposits were confirmed around the nasal corneal limbus.

pigmented lesion measuring $5 \mathrm{~mm}$ was first confirmed at the inferior nasal bulbar conjunctiva. The pigmented lesion at the nasal bulbar conjunctiva remained in place for a long time. When she everted her right lower eyelid at the age of 78 years, she noticed that the darkbrownish mass had become enlarged. Whether the large size suggested rapid growth was unknown. Therefore, she visited our hospital. Her medical history included coronary artery bypass grafting; her family medical history was unremarkable for any major disorders.

\section{Clinical Findings}

An initial examination revealed that the best-corrected visual acuity was 16/20 in each eye. Ocular motility, alignment, and pupillary response were normal in both eyes. No abnormal findings were observed in the cornea and sclera of either eye. The anterior chamber was of normal depth and was quiet in both eyes. Mild senile cataracts were present in both eyes. Intraocular pressure and fundus examinations were unremarkable for both eyes. Right ex- traocular findings by slit-lamp microscopic examination followed. The large, thick, dark-brownish mass extending from the inferior nasal semilunaris conjunctiva to the lower lacrimal caruncle measured 10 to $15 \mathrm{~mm}$ in diameter (Fig. 1a). The surface of the mass did not appear to be smooth (Fig. 1b). Transparent intralesional cysts, dilated feeder vessels, and ulceration were not observed. Movability of the large mass was confirmed not at the lacrimal caruncle but at the nasal plica semilunaris of the conjunctiva. A portion of the mass might have involved the inferior conjunctival fornix (Fig. 1a). Slight pigmentation could be observed at the nasal bulbar conjunctiva around the corneal limbus (Fig. 1c). T1- and T2-weighted magnetic resonance images of the orbit showed a high signal intensity, and images of the extraocular mass revealed an iso- to hyperintense signal. Moreover, magnetic resonance images could not demonstrate invasion of the ocular rectus muscle (Fig. 2). Based on the clinical features of older age, enlargement of the mass, less movability of part of the mass, the irregular surface, 
Fig. 2. Magnetic resonance imaging findings (T1 - and T2-weighted images). The giant mass showed a hyperintense signal on T1 imaging and an iso- to hyperintense signal on T2 imaging (yellow arrows).
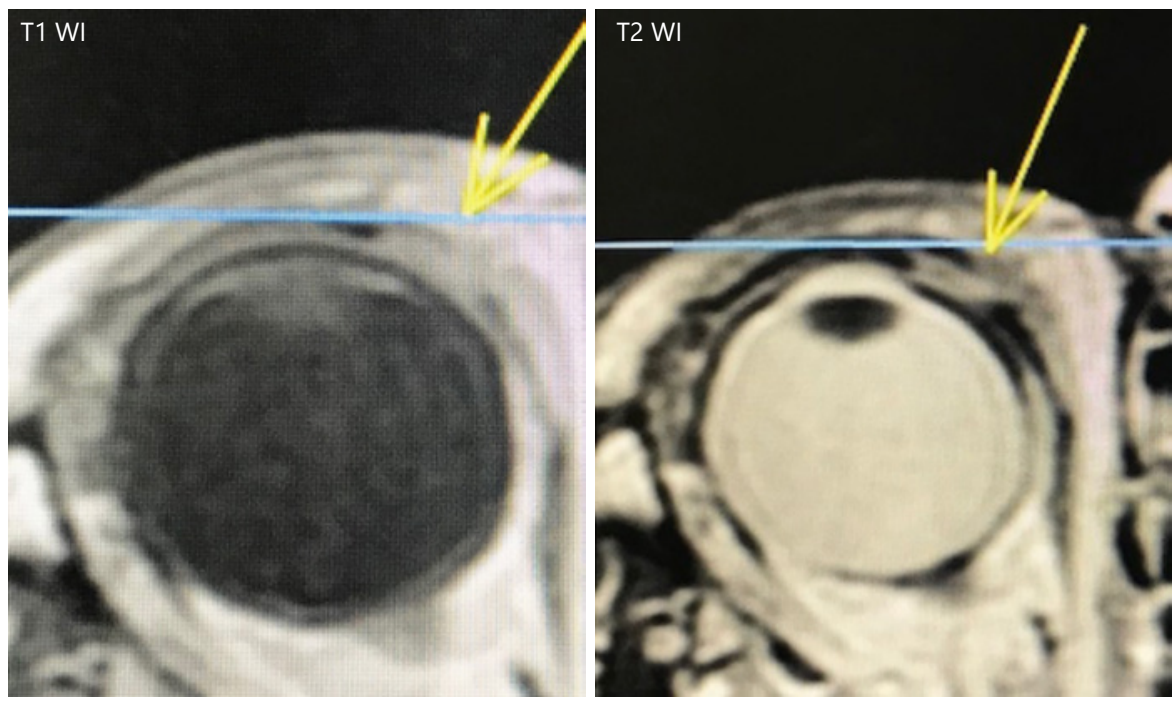

a lack of cystic lesions within the mass by slit-lamp microscopic examination, and rich pigmentation, we diagnosed this large mass as conjunctival malignant melanoma clinically, despite the lack of patient or family history of cutaneous melanoma. In April 2019, we performed a local excision of the mass combined with cryotherapy and application of $0.04 \%$ mitomycin without resection of the ocular rectus muscle or the use of reconstructive materials such as amniotic membrane. The majority of the mass was removed and submitted to pathology for definitive diagnosis. Slight melanin pigmentation remained at the corneal limbus and lower eyelid in her right eye (Fig. 3). Surgery was performed without complications. Ten months after resection, the conjunctiva in her right eye showed no development of tumor recurrence.

\section{Histopathological Findings}

The resected specimen was fixed in $10 \%$ buffered formaldehyde solution and processed for histopathological examinations. Hematoxylin-eosin (HE) staining and immunohistochemistry (IH) were performed. For IH, antibodies against S-100, melan-A, HMB-45, and $\mathrm{Ki}-67$ were used to investigate whether the mass was a malignant melanoma. Microscopic examination of a gross section stained with HE is shown in Figure 4a. The conjunctival epithelium showed thinning. Numerous nevus cell nests with abundant melanin and multiple mucous cysts lined by goblet cells were distributed in the subepithelial tissue of the conjunctiva (Fig. 4b, c). None of these lesions appeared to have malignant features, such as mitotic figures, atypical nuclei, a high nuclear-cytoplasmic ratio, or anisonucleosis. Moreover, pigmentation and atypical melanocytic hyperplasia in conjunctival epithelium were not confirmed. IH revealed positive S-100 and melan-A expression. S-100 expression was strong and diffuse throughout the specimen (Fig. 5a), while expression of melan-A was confirmed to be strong in melanocytic tissue (Fig. 5b). IH also revealed low immunopositivity for HMB-45 in the superficial layer (Fig. 5c). In contrast, immunoreactivity of Ki-67 (a proliferative index) was negative (Fig. 5d). Based on these histopathological findings, the large conjunctival melanocytic mass was diagnosed as a benign giant conjunctival melanocytic nevus.

Giant Conjunctival Melanocytic Nevus

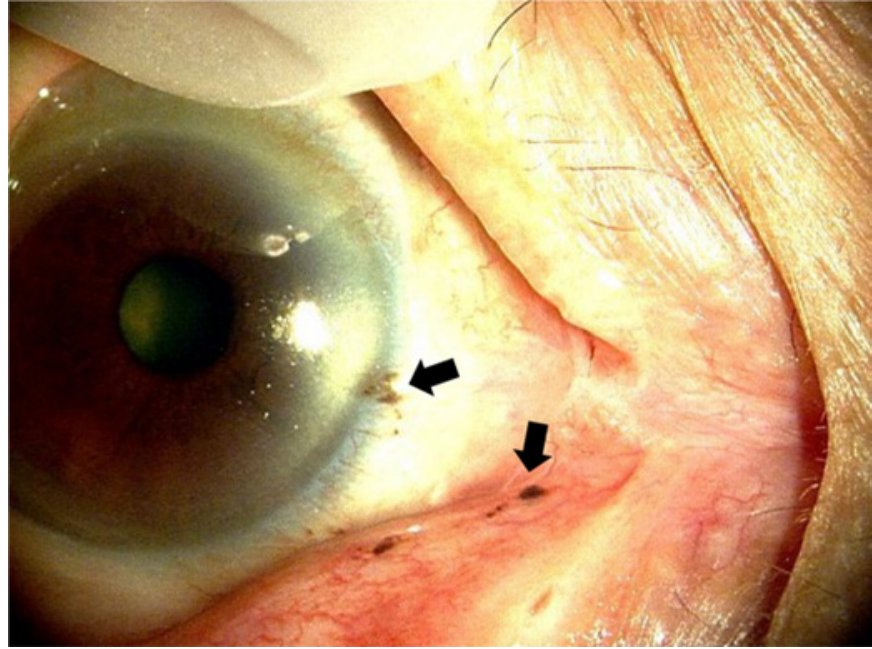

Fig. 3. Postoperative findings (postoperative month 10). The mass was resected completely and showed no recurrence. Slight residual pigmentation remained on the lower eyelids and corneal limbus (black arrows).

\section{Discussion}

The mean basal diameter of a typical conjunctival nevus is $4.1 \mathrm{~mm}$ [1]. In comparison, giant nevi have a mean basal diameter of $16 \mathrm{~mm}$ [3]. Among previously reported cases, the largest giant conjunctival nevi in the world and Japan have been 30 and $23 \mathrm{~mm}$, respectively [3, 4]. To our knowledge, the present case is the third largest conjunctival nevus to be reported. Giant conjunctival nevi represent approximately $5 \%$ of conjunctival nevus cases [3]. Therefore, the present case is rare.

Ocul Oncol Pathol 2021;7:97-102 
Giant conjunctival nevi can be confused with conjunctival malignant melanoma clinically [3-5]. Malignant melanoma is one of the most severe life-threatening diseases. Thus, differentiation between a giant conjunctival melanocytic nevus and a conjunctival malignant melanoma is very important with respect to management and treatment, as their prognoses differ dramatically $[1,6]$. The presence of characteristic features, including intralesional cysts, occurrence at a younger age, and stability over time, suggests a nevus rather than malignant melanoma $[1,3]$. In contrast, occurrence in middle-aged or older patients, protrusive lesions, rapid increases in pigmentation and/or size, ulceration, and a rich feeder vessel network strongly suggest malignant melanoma [6]. In the present case, the progressive findings of enlargement during adulthood (from $5 \mathrm{~mm}$ in childhood to $15 \mathrm{~mm}$ at 78 years), a lack of cysts by slit-lamp microscopic examination, and older age could be used to avoid clinically diagnosing conjunctival melanocytic nevi as conjunctival malignant melanoma. In particular, intralesional cysts strongly suggest conjunctival melanocytic
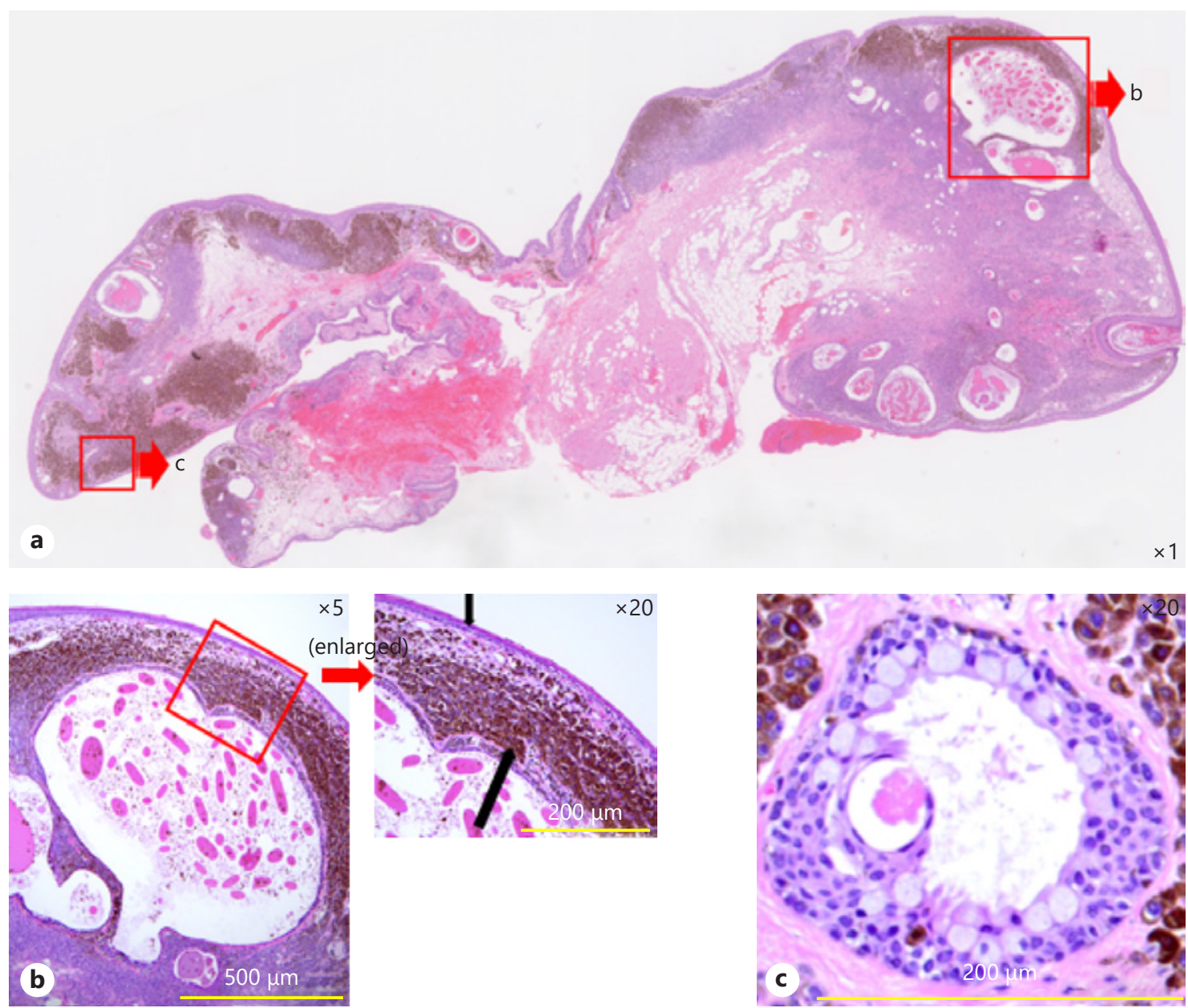

Fig. 4. Light microscopy findings of the mass with HE staining. a Gross appearance of the lesion $(\times 1)$. b Numerous nests composed of pigmented nevus cells were distributed at the subepithelial layer. Cysts consisting mostly of mucus were confirmed under thick pigmented nevus cell nests $(\times 5$ and $\times 20)$. c Small goblet cells lined the space around the mucosal cysts $(\times 20)$.

Fig. 5. Immunohistopathological findings of the mass. Red chromagen represented immunopositivity in each slides (S-100, melan-A, HMB-45, and Ki-67). S-100 and melan-A staining was diffusely and strongly positive (a, b). HMB-45 staining was positive in the subepithelial layer (c). In contrast, Ki-67 staining was negative (d).

(For figure see next page.) 

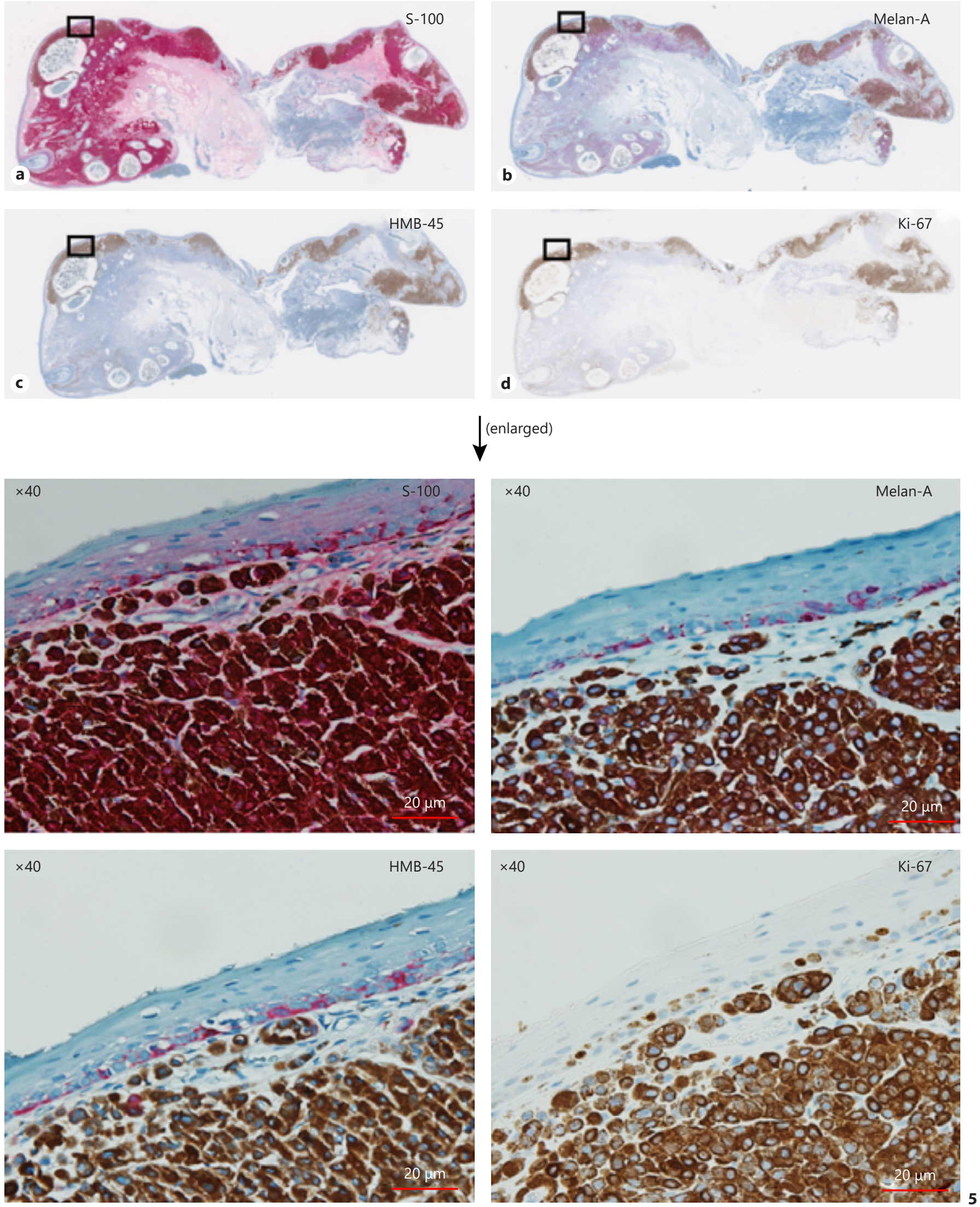

Giant Conjunctival Melanocytic Nevus

Ocul Oncol Pathol 2021;7:97-102 
nevi $[3,5,7]$. Recently, the use of anterior segment optical coherence tomography (AS-OCT) has been found to reliably detect intralesional cysts $[3,5]$. In the present case, ASOCT examination might have helped to predict the preoperative diagnosis of a conjunctival nevus. Performance of a clinical evaluation alone can lead to misdiagnosis. To make a correct diagnosis, histopathological evaluation must be performed. In the present case, $\mathrm{HE}$ and $\mathrm{IH}$ findings, which revealed multiple intralesional cysts, a lack of malignant cells, and negative Ki-67 expression, contributed to an accurate diagnosis of conjunctival melanocytic nevus.

A few previous reports about the potential malignant transformation of giant conjunctival nevi have been published $[1,8]$. However, only $3 \%$ of giant conjunctival nevi have been reported to transform to conjunctival melanoma $[1,8]$. Furthermore, the reported incidence of giant conjunctival melanocytic nevi undergoing such a malignant change is $<1 \%$ ( 1 case out of 32 ) [8]. In that case, malignant transformation occurred slowly over 23 years. Hence, it is important for such lesions to be subjected to long-term follow-up to ensure detection of transformation to melanoma.

In conclusion, giant conjunctival nevi can be diagnostically confused with conjunctival malignant melanoma. Considering the difficulty of preoperative diagnosis of giant conjunctival melanocytic melanoma, histopathological examination must be performed to ensure a correct diagnosis. Complete surgical resection is preferred to reduce the risk of transformation from a nevus to malignant melanoma. Even in the absence of recurrence, giant conjunctival melanocytic nevi require long-term monitoring.

\section{Acknowledgement}

The authors thank the Department of Pathology and Laboratory Medicine, Kansai Medical University for making histopathological tissue section.

\section{Statement of Ethics}

The patient has given informed written consent for the publication of the clinical and histopathological photographs and the medical findings. No IRB approval was required because this was a case report that conformed to the Code of Ethics of the World Medical Association (Declaration of Helsinki) and obtained informed patient consent.

\section{Conflict of Interest Statement}

The authors declare that there are no financial or other conflicts of interest.

\section{Funding Sources}

None.

\section{Author Contributions}

Hidetsugu Mori has been involved in the manuscript writing, final critical review, and submission. Kanji Takahashi has been involved in patient management and manuscript editing.

\section{References}

1 Shields CL, Fasiuddin AF, Mashayekhi A, Shields JA. Conjunctival nevi: clinical features and natural course in 410 consecutive patients. Arch Ophthalmol. 2004 Feb;122(2): $167-75$.

2 Shields CL, Demirci H, Karatza E, Shields JA. Clinical survey of 1643 melanocytic and nonmelanocytic conjunctival tumors. Ophthalmology. 2004 Sep;111(9):1747-54.

3 Shields CL, Regillo AC, Mellen PL, Kaliki S, Lally SE, Shields JA. Giant conjunctival nevus: clinical features and natural course in 32 cases. JAMA Ophthalmol. 2013 Jul;131(7):85763.
4 Tomita M, Goto H, Muramatsu R, Usui M. Treatment of large conjunctival nevus by resection and reconstruction using amniotic membrane. Graefes Arch Clin Exp Ophthalmol. 2006 Jun;244(6):761-4.

5 Tóth-Molnár E, Vizvári E, Skribek Á, Vörös A. Giant Conjunctival Nevus in a 12-YearOld Child. Case Rep Ophthalmol Med. 2017; 2017:8414352.
6 Shields CL, Markowitz JS, Belinsky I, Schwartzstein H, George NS, Lally SE, Mashayekhi A, Shields JA. Conjunctival melanoma: outcomes based on tumor origin in 382 consecutive cases. Ophthalmology. $2011 \mathrm{Feb}$; 118(2):389-95.e1-2.

7 Folberg R, Jakobiec FA, Bernardino VB, Iwamoto T. Benign conjunctival melanocytic lesions. Clinicopathologic features. Ophthalmology. 1989 Apr;96(4):436-61.

8 Gerner N, Nørregaard JC, Jensen OA, Prause JU. Conjunctival naevi in Denmark 19601980. A 21-year follow-up study. Acta Ophthalmol Scand. 1996 Aug;74(4):334-7. 